\title{
So now you're ready to play - but with what? A system to encourage playful exploration
}

\author{
Jon M Pearce \\ The University of Melbourne \\ Parkville \\ Victoria, Australia \\ j.pearce@unimelb.edu.au
}

\author{
Sofia Pardo \\ The University of Melbourne \\ Parkville \\ Victoria, Australia \\ miriamp@pgrad.unimelb.edu.au
}

\begin{abstract}
One of the challenges in encouraging learning through play is to design online environments that are playful yet not distracting; that encourage interaction and exploration with specific learning goals while minimising the distraction of 'bell and whistles'. This paper presents the application of theories of play and flow in the design of a highly interactive and playful online system that has been used as a research tool to explore students' exploration and interaction within such environments. We present applications of the system to several different contexts such as choosing university subjects, and solving puzzles. We describe a particular experiment that helped us to identify deficiencies of the system, discuss students' interactions with it, and describe follow-up work that is both currently underway and also planned for the future.
\end{abstract}

\section{Categories and Subject Descriptors}

H5.m. Information interfaces and presentation (e.g., HCI): Miscellaneous.

\section{General Terms}

Performance, Design, Experimentation, Human Factors.

\section{Keywords}

Exploration, mindful engagement, search, play, flow, multimedia.

\section{INTRODUCTION}

One of the powerful effects of play on learning is that you do not necessarily appreciate that you are in fact learning. As you interact with artefacts on a screen, people online, or objects in a room, you can become so immersed that you fail to notice all of the mental connections that your brain is making. You can become so engrossed in the playful environment that the learning just happens. Or does it? At other times you might become engrossed in the play, and just - play! Sure, some learning might take place, but it might not relate at all to the intentions of the designer of the environment. You might learn to play the game, but not necessarily learn the strategies or understand the concepts.

Hence we see a distinction between playful environments that happen to engender learning and playful environments that are designed for learning.

This paper addresses the issue of using playful environment as a means of attracting people to a task, but then maintaining their interest in that task, and learning from it. We describe a playful online system that has been designed to be attractive to users and evoke persistent exploratory behaviour. We explore how users interact with the system and whether it maintains their interest through its playfulness, while encouraging them to focus on the task at hand.

\section{BACKGROUND}

This research grew from earlier research that studied how flow relates to learning $[10,11]$. The concept of 'flow', that highly engaging and enjoyable experience characterised by people losing the sense of time as they become immersed in an activity, has been studied in many contexts. Research has investigated flow in areas of sport, entertainment, Web design, eCommerce and many others. On the one hand, the focus can be one of obtaining greater satisfaction from everyday life - engaging in more activities that might result in a sense of happiness or greater fulfilment [5]. On the other, flow has been proposed as an experience that might be desirable for more predetermined and specific outcomes, such as creating a learning environment that is highly supportive of students' learning.

What is it about the nature of flow that suggests it as a desirable state for such a diverse range of activities from computer games to learning physics? The answer, at least for these two extremes, is quite clear. Game designers have developed a talent for designing rich, playful environments that captivate minds for hours on end; these are surely the envy of instructional designers. Not only is the game-player captivated, but he or she comes back time and time again to enjoy the experience with scant regard for the hours that fly past. If only this could be the case for learning software! Is it not the holy grail of educational software to grab students' attention through activities that are enjoyable, support learning, and have the students craving more?

The difference between these two software genres is clear: one aims simply to provide a challenging and enjoyable experience that is as addictive as possible, whilst the other adds to that the aim to teach specific learning outcomes. Whereas merging these two aims - possibly through our understanding of play and flow - 
might seem straightforward, their mutual compatibility is not so certain. The intrinsic motivation offered by a highly engaging game might result in the user learning much about the gaming world, but an overly animated educational package might see a student focusing more on the alluring screen interactions than on the content that is intended to be learnt [13].

This focus on task is sometimes described as 'mindfulness'. A 'mindful state' is used to describe the performance of a particular task in which the state is characterized by non-automatic, metacognitively guided mental processes that require mental effort [18]. A mindfully engaged learner will display higher order thinking behaviours such as strategic thinking, self-monitoring, evaluation of responses and reflection [20]. In contrast, a mindless state implies a trial-and-error approach that relies mostly on automatized processes. This state is evidenced by lower order thinking activities such as mastery of procedures, browsing and information seeking. Our challenge with playful environments is to design them with the outcome of promoting mindful interactions.

There is an additional factor in a learning environment. It matters what is learnt. Typically, in a learning package, there is a preset agenda and the aim is that students learn specific content, concepts, procedures, processes, etc. This assumes that either students know that they have learnt adequately, or they are in some way tested, or given feedback, on their knowledge acquisition. Nevertheless, there may be much learning taking place that is not tested and not recognised. For example, with software designed to help students learn physics, the acquisition of specific concepts maybe a desired outcome. However, whilst doing this, students may acquire or reinforce many other ideas such as interpreting graphs, working with numbers, use of technical language, etc. Such 'incidental learning' is rarely investigated in the research literature as it is very hard to identify. In addition, it is very hard to ascertain the veracity of such learning; how often do inappropriate ideas form in the minds of students?

So the challenge we have is this: how do we strike a balance that provides a highly playful environment, that attracts and maintains a student's attention, and at the same time ensure that the student's focus is directed towards the tasks to be learnt? And how do we deliberately design for this?

\subsection{Concepts of flow and play}

'Flow' describes a state of complete absorption or engagement in an activity [5]. It is a highly enjoyable state. The study of flow crosses many discipline boundaries including Human-Computer Interaction [21] and instructional design [12].

Chen, Wigand and Nilan [4] grouped the elements of flow into three stages: antecedents, experiences and effects. The first stage, antecedents, comprises:

- a clear set of goals;

- timely and appropriate feedback; and

- a perception of challenges that are well matched to the person's skills.

The second stage, experience, comprises:

- a merging of action and awareness;

- a sense of control over the activity; and

- concentration.
The final stage, effects, describes the individual's inner experience:

- loss of self-consciousness;

- time distortion; and

- a feeling that the activity becomes worth doing for its own sake ('autotelic').

A fundamental feature of a flow experience is an activity that offers a person an appropriate balance of challenge and skills. If the challenge is too great for the person's skills, he or she will become frustrated, lose interest and stop flowing. If the challenge is too low, the activity ceases to be interesting and boredom can result. The goals for the activity must be clear and provide quick and unambiguous feedback in order to promote flow. The resulting focused concentration can lead to a "merging of actions and awareness' in which 'one does not have enough attention left to think about anything else' [5]. Other common features of flow are a distorted sense of time, usually seeming much shorter than it actually is and, because of the deep concentration, the person can lose the awareness of self that often intrudes into one's consciousness. The combination of these effects leads to a feeling of intrinsic enjoyment - an autotelic experience.

It is interesting to compare the concept of flow with that of 'play', which has been described as the 'flow experience par excellence'[5]. Playfulness is difficult to define and measure [2, 16] but is often used to represent a person's subjective experiences that involve perceptions of pleasure and involvement [19] which can enhance learning [9]. Whereas playfulness can contribute to creativity [5] it can also have negative consequences such as lengthening task completion times due to exploratory behaviour [5]. Webster, Trevino and Ryan [21] note that playfulness may result in longer times for task completion, even to the extent of neglecting key tasks.

We can try to draw a distinction between the concepts of play and flow. Webster, Trevino and Ryan [21] use flow as a theoretical foundation on which to explore playfulness with the use of technology in the workplace. Whilst they do not clearly distinguish between the concepts of flow and playfulness, their experiments imply that they regard playfulness as being affected by perceptions of flexibility and modifiability of the software, experimentation and a sense of enjoyment that leads to further voluntary interactions.

Play is regarded as an example of an optimal flow experience which can relate to learning [15]. Rieber and Matzko [16, 17] consider play in a physics learning context and define serious play as being an intensive and voluntary learning interaction consisting of both cognitive and physical elements. Flow activities also often involve physical elements, for example, in chess, rock climbing, surgery [5]. But there are also flow activities that require no such physical interaction with an object, for example: a conversation, reading a book, watching a movie.

Given the common features of play and flow, this suggests that a flow activity might be regarded as being either playful (e.g. chess) or not (e.g. surgery) distinguished by the motivation and goals of the person - for leisure or for work. Rieber [16] sees play as a means for understanding the relationship between learning and motivation in a holistic manner and concludes that learning and motivation are inseparable with motivation being the more important of the two. He describes a rewarding learning experience as being satisfying and enjoyable. Whether being 
carried out by a child or adult, he regards the best word to describe this as 'play'.

The distinctions in meaning between flow and play are subtle but significant. We can consider flow to extend the concept of playfulness [5, 6, 9] by incorporating the extent to which users perceive a sense of control, focused attention and cognitive enjoyment [21]. Flow fits well with a constructivist model of learning by adding these three attributes beyond those of play [21]. The tight link between goals and feedback drives and supports learning activities. Flow suggests a greater sense of purpose and intention, whereas play emphasises enjoyment and activities that might lack direction and outcome [7].

To encourage exploratory user interaction in the research described in this paper, we drew on our understanding of the concepts of 'flow' and 'play' in aspects of the design. That is, we aimed to design an environment in which the users felt well in control, had clear goals, and received timely and appropriate feedback as to their success in achieving these goals [3]. However, we also wanted the environment to be interesting and 'playful' - often achieved by rich interactions or a sense of novelty. In addition, it was important that the user had a sense of purpose and intention. We proposed that this combination of interest and playfulness in a supportive environment should encourage the users to explore, to consider 'what-if' questions, and to feel a sense of immersion with the experience.

\subsection{The challenge of designing for flow \& play}

Two important factors in informing the design of interactive systems are 'engagement' and 'positive affect'.

Flow theory reminds us that 'engagement' is dependent on the user feeling in control, having prompt and appropriate feedback to his or her actions, and perceiving a degree of challenge that is sufficiently greater than perceived skills. However, that is only part of the story. In a learning context, where often the task is not self-motivated but is assigned by others (teachers, lecturers), there is a need to ensure that the student's engagement is focussed on the learning task itself and not just the affective aspects of the visual or audio interface. This presents the designer with the challenge to manipulate a delicate balance between the learning task and interactions with the user interface. Should the task be too challenging for an individual student, then that student might readily shift focus to distractions such as a cute window-zooming effect or sliding the scales on a graph to see the elegant re-scaling of data. This is not such an issue in a gaming environment as the user is likely to be more self-directed and has the overarching goal of having fun rather than attaining outcomes pre-set by others.

'Positive affect' refers to the positive emotional state that a person is left in after a flow experience. During flow a person's mind is fully occupied by the experience, leaving little room for being conscious about the experience. However, after the experience has ended, a person may reflect and become aware of a feeling of happiness [5]. The importance of this in interface design is that people like to do things that make them happy. When people make comments such as 'I don't enjoy physics' or 'I don't like interacting with that particular Web site' they are not reflecting on positive or happy experiences. If experiences can engender more positive memories in the user, then they are more likely to return and participate on subsequent occasions.
The aim of the study described here was to design engaging experiences that leave the user with positive feelings and hence a tendency to persist with the task and be prepared to return at later times. The balance between engagement with playful screen artefacts and engagement with tasks had to be carefully balanced.

\subsection{Balancing challenge and skills}

One condition for flow is an appropriate balance of perceived challenges and skills. But how do you accomplish that when the skills of the user are unknown to the designer? A skilled teacher achieves this in dialogue with a student by recognising the student's difficulties and responding by adjusting her own words, or varying the learning task, to accommodate accordingly. Intelligent adaptive learning environments attempt to do the same.

An alternative approach, and one often employed by default, is to let the learner or user become part of a feedback loop and hence adjust their own challenges. This is done in many situations by encouraging the use of 'what if' questions. 'What will happen if I change the value of this parameter?', or 'what if I change my response to this earlier question?'. If such options to explore are clear and easy to implement, then users can adjust their challenges in response to their reaction to the tasks. The design challenge here is to make these options easy for users to recognise and implement, and to make the consequence of them (feedback) immediate and unambiguous.

In this research we have included the user as a major player in the feedback loop of a system hence increasing the chance for flow by providing a moderating or stimulating effect on the challenges being presented.

The above discussion has shown how the ideas behind flow and play have been drawn upon to help in the conceptual design of an online system. To be successful, the system needs:

i) to have a highly playful interface;

ii) to present challenges to the user; and

iii) to incorporate the user as part of a feedback loop that supports a 'what-if?' style of exploration.

\section{CONCEPTUAL DESIGN OF A SYSTEM}

In designing a system with which to explore ideas of play, flow and exploration, we chose the task of university students selecting subjects for their degree. At the university in which this study was conducted, students are required to take one quarter of their subjects (i.e. one subject each semester) from outside their main discipline area. These are referred to as 'breadth' subjects. Designing a system to facilitate this task was seen as an appropriate choice of task for three reasons:

- the task was open-ended allowing students to explore for as long as they liked;

- the task was challenging in that there were 2300 subjects to choose from, most of which the students would have little if any prior knowledge;

- there was an opportunity for user feedback since the students would have some idea of which subjects presented were appealing to them and could hence react to the output of the system. 
We carried out a pilot study [14] with five students to help understand how students, at the time, went about making subject selections using the university's online handbook. This showed the student selection process to be a strongly hierarchical one: a student would link to a Faculty page in the handbook, choose an interesting looking department, look at the subjects for the appropriate year of study, choose from that list of subjects, read the handbook description, then select (or not) the subject, and then return to the Faculty page and start again.

In a focus group we discussed other ways of carrying out this task. We proposed a set of sliders that might control the ranking of a list of subjects using 'meta' descriptors relating to students' interests. The proposed meta-description scales were:

\section{Relating to discipline of study:}

\author{
pure/applied; \\ qualitative/quantitative; \\ light/dense on imagination \& creativity; \\ factual/conceptual.
}

\section{Relating to learning experiences:}

\author{
hands-on/brains-on; \\ exploratory/directed; \\ working alone/with others; \\ oral communication light/dense.
}

The focus group reacted positively to these suggestions. They told us that the system would need to be playful to hold their interest in the task, and dynamically interactive. They made several suggestions as to its features, and, interestingly, they asked the question "who would decide how each subject rates on the scales: students or staff?". This is an interesting issue to which we return later.

\section{IFISH: A SYSTEM FOR EXPLORATION}

We took the findings from the pilot study and designed an online interactive playful system to assist students in choosing subjects. The system had the following broad goals:

1. The system should have playful affordances in order to encourage sustained exploration;

2. It should provide immediate, real-time feedback in response to the user's input in order to encourage 'what if?' style interactions;

3. The user's input should be based on meta-data relating to the user's own experience or preferences, rather than knowledge of the content that the system is allowing users to explore (this relates to the fact that, in this particular context, students would know very little about the range of subjects that they could choose from).

The output of the system would be a list of appropriate subjects, ranked according to how well each subject matched the user's preferences. This is not unlike a 'recommender system', common in sites such as Amazon.com, Last.fm.com and Pandora.com. (For a technical review of recommender systems, see [1].)

However, in this system, rather than narrowing a search down to a few 'hits', our aim is to provide sufficient stimulus for the user to change an input, observe the outcome, reflect on the effect of the change, and then make further changes. That is, when the user makes a small adjustment to an input slider, the immediate, realtime, animated change presented on the screen might prompt the user to think 'Oh - that was interesting. I wonder what would happen if I moved this slider even a little more in this direction?'.

A screenshot of the completed application ${ }^{1}$ is shown in Figure 1. The application was produced using Adobe Flash with extensive use of XML files to control data, behaviours and settings. Subjects are represented by the three columns of labels (which we refer to as 'fish') in the upper left quarter of the screen; one column for each year level. These subjects are ranked by how well they match the combination of slider values visible at the bottom left. A 'wish list' area at the top right allows students to drag subjects to keep for later reference. A list of university faculties is located to the right of the sliders allowing individual faculties to be excluded from the search.

The behaviour of the system is as follows. As the user changes the sliders, the subject titles ('fish') 'float' up and down. Students can drag fish to the top-right area in order to keep them for later. Clicking on a fish will expand it and display a description of the subject as presented in the university handbook. Students can use the set of checkboxes in the centre lower screen to remove subjects in particular faculties. They can delete subjects that they have no interest in and others will 'float up' to replace them. All student activities are logged to a server during operation.

The name iFISH was derived from interactive Foraging In the Subject Handbook. It gave an appropriate flavour of 'fishing around' for results.

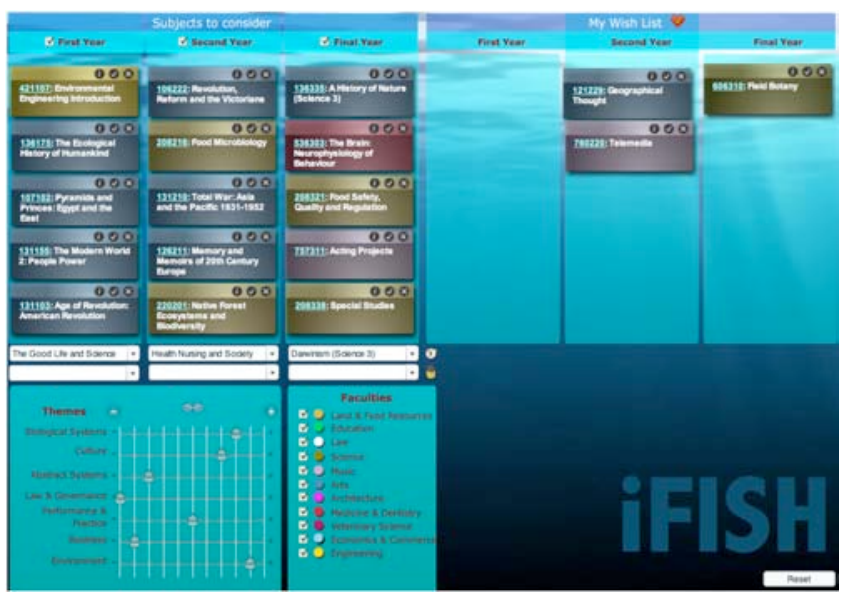

Figure 1: A screen-shot of $\boldsymbol{i F I S H .}$

The category labels on the sliders (not clearly visible in the figure) are not the same as those presented in the pilot study described earlier in this paper. The reason for this comes back to the issue of who determines the ratings for each subject. It was not feasible to tag each of the 2,300 subjects manually. We opted to try an automated tagging based on the 200-word subject descriptions listed in the online handbook. We tried various linguistic analysis techniques to seed a clustering process based on the desired categories, but without great success. (A fuller description of this process can be found in [14]).

1 The actual application can be accessed from http://disweb.dis.unimelb.edu.au/staff/jonmp/projects/iFISH/ifish.html 
We ended up identifying clusters of subjects from which we derived the following seven labels:

\author{
Biological systems \\ Culture \\ Abstract systems \\ Law and governance \\ Performance and practice \\ Business \\ Environment
}

These were far from ideal and did not have the bipolar nature of the original suggested categories. Nevertheless, given that this system was built as a research tool rather than an application for serious use, they provided an adequate means through which to drive the system and observe the animated effects on the fish.

\section{EXPLORING THE USE OF IFISH}

\subsection{Research Questions}

Five research questions were formulated, each of which related to a particular construct as shown in brackets below:

1. Does having the opportunity to enter individual preferences (through the use of sliders) encourage users to reflect on their preferences? [Reflection]

2. How does the playfulness of the application (e.g. animation, sliders) influence the user's experience? [Playfulness]

3. Does iFISH encourage breadth of discovery? [Novelty]

4. Do users engage mindfully when interacting with iFISH? [Mindful engagement, cognitive load]

5. How are the results provided by iFISH perceived by the users? [Outcome satisfaction]

\subsection{Data collection method}

\subsubsection{Objective:}

An experimental design was implemented in order to reveal users' perceptions and experiences while using iFISH to search for breadth subjects. These perceptions were gathered by contrasting iFISH with use of the current university handbook.

\subsubsection{Participants:}

Seven first year students (all male) participated in the experiment. All participants had previously used the university handbook but were new to iFISH.

\subsubsection{Experiment set-up:}

All participants were given four tasks to undertake with iFISH and the handbook over a period of 1.5 hours. The order in which these tasks were carried out was alternated to minimize order effect. Immediately after completing two tasks with iFISH and with the handbook participants were asked to answer three questions relating to: time-on-task versus time driving the application; and cognitive load. Each of these questions was answered with a five points Likert scale. This was followed with a questionnaire consisting of 16 statements relating to their interaction with the system, to which they were asked to answer using a five point Likert scale. A debriefing session followed.

\subsubsection{Data analysis method}

The analysis of the data comprised three stages:

1. Post-task questions and questionnaires were aggregated and an average was calculated in order to identify any tendencies.

2. Computer log files were reviewed in order to record the time spent on each task, the number of subjects students clicked on to expand and read details and, in the particular case of iFISH, the amount of slider movement carried out.

3. The researcher's notes were also reviewed in order to provide an enriched view of participants' experiences and opinions.

\subsection{Findings}

Findings are presented in this section following the research questions stated previously.

1. Does having the opportunity to enter individual preferences (through the use of sliders in iFISH) encourage users to reflect on their preferences? [Reflection]

Users' responses to the questionnaire showed that most considered that the sliders prompted them to reflect on their preferences. However, they were mostly neutral with respect to being prompted to think about choosing subjects in a different way. Although nearly all users found the sliders easy to use, most of them wished to have a search box to be able to enter keywords.

"Didn't allow me to narrow down ... but it didn't know what I wanted that would be really helpful" [Participant 4]

This finding illustrates that many users were eager to narrow down their search as opposed to spending time exploring with the sliders. It can be argued that most users followed a 'question answering' strategy when completing the tasks provided. This was further confirmed by most users trying to de-select as many options as possible from the list of faculties so that a more limited number of subjects would be displayed.

Most users decreased their use of sliders from one task to another. Only two users increased their use of sliders and one remained the same. This may be the result of users gaining proficiency with the sliders as they progressed with the tasks. Furthermore, after setting the sliders according to their preferences, some users did not consider it necessary to alter them but were more interested in seeing a larger number of subjects that matched the original set of preferences.

2. How does the playfulness of the application (e.g. animation, sliders) influence the user's experience? [Playfulness]

The task of searching for subjects was not in itself considered a tedious task by most users; however most of them enjoyed using the handbook more than iFISH to accomplish it.

Regarding the animations in iFISH (e.g. sliders, fish movements) some users perceived that the animations hindered the task as they wanted to access the information quickly. Although some users acknowledged that iFISH was more visually pleasing, it did not appear to make the task easier or more enjoyable.

Some users commented that the look and feel of iFISH was not consistent with what was expected of a handbook, to be 'professional and clean'. iFISH was perceived on the other hand as 'for children'. It can be argued that iFISH successfully 
conveyed a sense of playfulness, but this was considered by one participant to be inappropriate and unnecessary for the task at hand:

“... it is not suitable for this purpose, it is only a search engine. It is a nice way of presenting but it doesn't need it because it is the content that matters. No need to be engaging or visually appealing." [Participant 4]

\section{Does iFISH encourage breadth of discovery compared to} the handbook? [Novelty]

Breadth of discovery was determined by the number of subject 'fish' expanded by the users in order to read the subject description. In most cases users expanded more subjects in iFISH than they navigated to using the handbook. However, it is important to bear in mind that in iFISH the range of subjects displayed is much broader than in the handbook, since in the latter breadth subjects are filtered to just those subjects that the students (according to their course and level) can actually take.

Users did not broaden their interest in subjects when using iFISH despite the fact that they were exposed to a larger number of subjects across faculties. It can be argued that users met their expectations with respect to the type of subjects they were likely to find in particular faculties.

\section{Do users engage mindfully when interacting with iFISH? [Mindful engagement, cognitive load]}

It was anticipated that iFISH would reduce the cognitive load of the task, hence encouraging mindful engagement by (1) allowing users to enter their preferences and immediately see the resulting subjects on the same screen, (2) navigating seamlessly across faculties without the need to follow a hierarchical structure and (3) having the possibility of pre-selecting subjects into a wish list for later consideration. In contrast, the handbook requires users to enter their profile (year level and course), their preferences (areas of study) and submit a query on the first screen, which then results in a list of potential subjects.

Users found the selection of breadth subjects using iFISH more difficult than when using the handbook. However when prompted to determine the mental effort involved in the task they considered that both required a similar effort. This suggests that the decisionmaking task in itself involved a similar mental effort in both cases, while the interface to the task was much more demanding in iFISH than in the handbook. Most users considered they spent more time working with the interface in iFISH than with the actual task.

It can be concluded that the cognitive load associated with the task was similar in both the handbook and iFISH, while the cognitive load associated with the interface was considerable higher in iFISH. The users' perceptions may be influenced by their familiarity with the handbook, which they had used previously for their own selection of breadth subjects.

\section{How are the results provided by iFISH perceived by the users? [Outcome satisfaction]}

Most users were not satisfied with the results displayed by iFISH. They felt they would not rely on the results to make their decision and were unsure they were going to find the most suitable subjects. On the other hand, they were more confident and satisfied with the results yielded by the handbook. The majority of users wanted to be shown ALL possible subjects they were able to do as breadth subjects (about 200 subjects for these students), as opposed to the top ones ranked in iFISH as the best match for the preferences they entered. Users tended to delete subjects from the iFISH list as a strategy for 'scrolling down' the list to see what subjects were ranked below those visible on the screen. Some users commented that they preferred to scan through a larger list of subjects than to be given small groups of subjects that gave them a sense of their search being incomplete.

“... there is a feeling of closure in the handbook, and that's what you are after when looking for subjects. You feel that you found all possible choices that best suited you”. [Participant 3]

In addition, the dissatisfaction with the results given by iFISH was exacerbated by the fact that iFISH did not filter those subjects that students were not allowed to do, due to their course or level, hence showing an extensive number of subjects that may have been of interest but not viable. This resulted in frustration as users considered there was no point in seeing suggested subjects that they were not allowed to do.

\subsection{Discussion on experiment}

The information gleaned from the participants in this study does not read like a glowing endorsement for an iFISH-style interaction for choosing university subjects. However this is not necessarily the case, and there is much to be learnt from the results presented. Since this was qualitative research carried out with only seven participants, the outcomes can in no way be considered as conclusive.

\subsubsection{Limitations of the experiment}

Lack of playfulness. The performance of the system was too slow to drive an immediate animated change to the fish displayed. There was a lag of 2 or 3 seconds between moving a slider and seeing the resulting changes on screen. This was due to the extensive calculations required to match user changes to subject attributes. The significance of this is that it reduced significantly the likelihood of a student moving a slider slowly, whilst watching the changes, and then deliberately nudging it one way or the other in anticipation of what the effect might be. In other words, it reduced the impact of 'what if?' strategies. This immediacy of feedback is essential to flow and we suggest is also important in achieving a satisfying play experience. Whereas some play experiences might benefit from promoting in the user a sense of anticipation, in a task such as the one presented here, this tends to lead to frustration. A later version of the software ('gFISH') has removed this lag (described later).

Inappropriate slider categories. As explained earlier, the subjects were not manually tagged according to predetermined attributes, but rather an automated linguistic analysis was carried out to determine slider categories and subject weightings. The nonoptimal nature of these labels did not really enable us to explore how students would respond to this task based on their personal preferences.

We have subsequently set up a system with a smaller set of subjects, using the newer software gFISH, in which the subjects have been manually tagged. This system provides immediate, real-time responses with fish gliding around the screen as the user moves the sliders. All twenty-eight subjects are visible on the screen at all times, and we have also implemented a feature to 
allow students to contribute their opinion to the weighting of tags as they use the system. Figure 2 shows this new system.

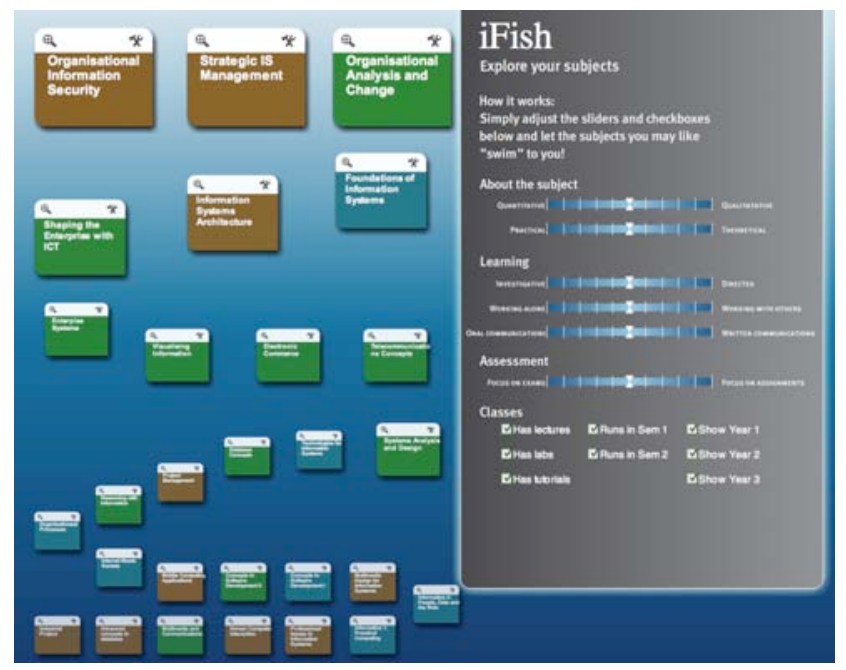

Figure 2: gFISH showing 24 subjects.

Figure 3 shows an enlargement of the control panel for this version, including the sliders, their labels and other check-box options.

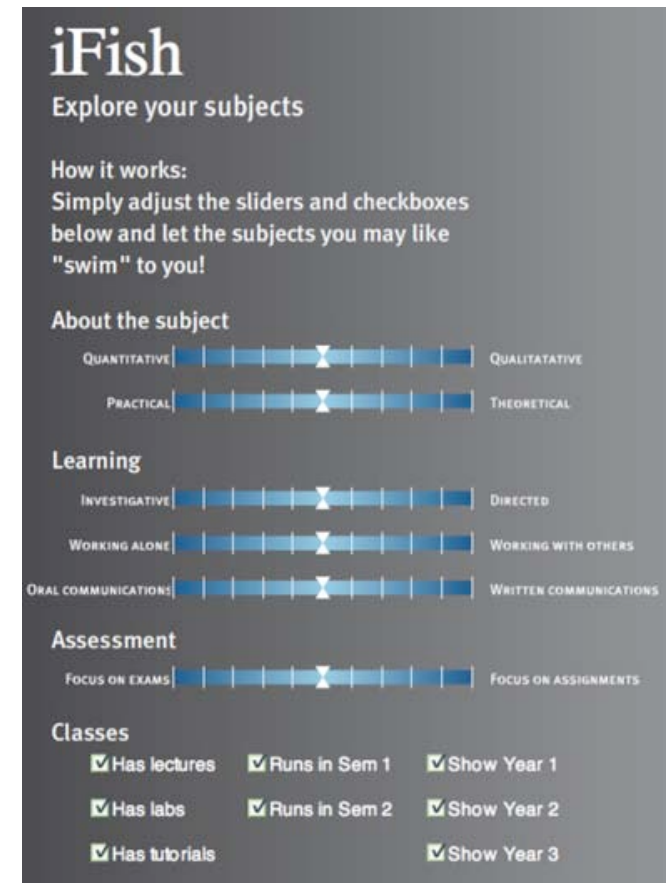

Figure 3: gFISH control panel.

Nature of the task. It was clear from the students in this study that they regarded the task of searching for subjects as a structured one that they had to work through and complete, rather than a more open-ended exploratory one that they might 'wallow' in for a while before making final subject choices. Despite encouraging exploration, students remained interested in narrowing down their search. In a sense we provided them with an unfair comparison. The requirement for breadth subjects was in its first year at the university and hence there were only about 200 subjects to choose from. The university had set up a new handbook site that presented these in a structured fashion making it easy to browse all subjects on one web page: students could click on a study area (e.g. environment and natural resources) and be taken to a section of the list showing about 50 choices. By contrast, iFISH was always working with 2,300 subjects unless the students 'turned off' various faculty areas in which they had no interest. Now that the breadth system is in its second year, there are many more subjects for students to choose from. A commerce student, for example, would see about 1200 subjects in total, and even when choosing only one subject year-level, that could include 500 subjects. We do not yet know the impact that such a large number of subjects will have on the use of the existing subject handbook.

Style of interface. It was also interesting to see comments from some students about matching the look and feel of the interface to that of the task. We only had a small group of participants, but at least some felt that a more professional look was warranted, rather than a playful one, for this task. These were university students, yet the interface was not 'childish' in its playfulness. This raises interesting questions about students' perceptions of play and playful interfaces in learning generally.

Personal preferences. Findings from this experiment raised the issues that prior experience and personal preferences may influence the way we approach open-ended or structured tasks. Therefore, when trying to encourage exploration an outcomedriven mindset or task can become a hurdle. As suggested in the literature, exploratory search is often iterative, takes place over longer periods of time and aims to maximize the number of possible relevant objects rather than minimize the number of possibly irrelevant ones [8].

\section{ONGOING WORK}

In order to use a system like iFISH to study playful interactions, engagement and exploration, we need to implement several changes. The first is to choose an appropriate task that can lead to extensive exploration. The second is to manually 'tag' the data so that we can use categories for sliders that enable users to genuinely express their personal preferences. Finally we must ensure that the system runs fluently and with immediate response.

As a spinoff from the iFISH development we have created gFISH (' $\mathrm{g}$ ' for generic). This is a highly configurable system allowing us to define data, tag weightings, behaviours and interface elements by editing simple XML files. This allows us to fairly quickly set up systems in which outputs are ranked according to user preferences. We have constructed quick examples such as choosing a camera to purchase, preparing a museum visit and choosing subjects within a single department.

These can be found on our project page: disweb.dis.unimelb.edu.au/staff/jonmp/projects/iFISH/ifish.html

An interesting example of using gFISH has been set up as an online experiment by a student currently completing a Masters degree. Ruud Knieriem (Utrecht University) has studied how users interact with engaging systems and how features of the system impact on engagement. His use of gFISH involved creating puzzles in which users explore sorting geometric shapes on the screen according to parameters such as number of sides, colour, or label. He has also set up gFISH to present a picture divided into sixteen parts that are scrambled on the screen (Figure 
4). The user's task is to reconstruct the picture using three unlabelled sliders or steppers.

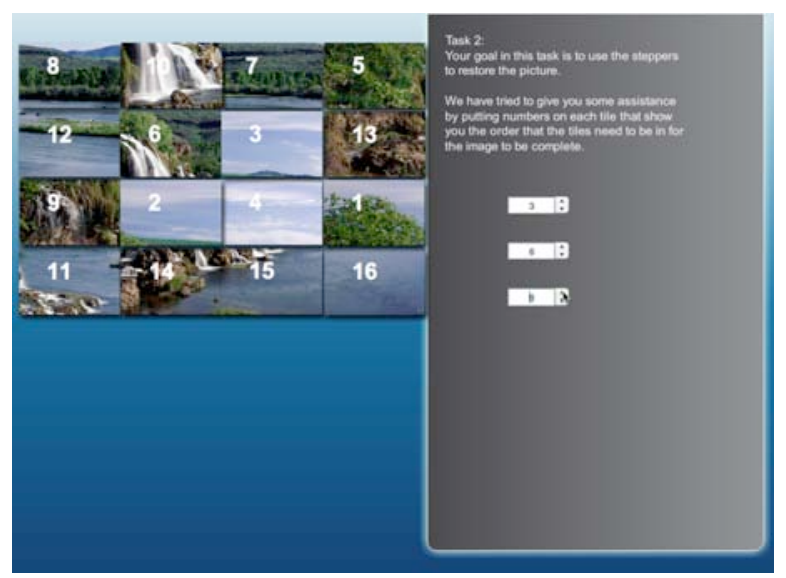

Figure 4: Using gFISH with steppers to re-construct a picture.

Findings from this experiment relate to how the use of sliders and steppers relate to control and task performance.

A further playful iFISH-style system is being developed to enable exploration of learning styles and preferences on users' paths through learning resources and how following these paths might impact on learning outcomes.

\section{CONCLUSION}

This paper has presented the design philosophy of a playful, interactive system (iFISH) that was designed as a vehicle for exploring playful interaction and exploration. The particular task chosen for this system was perceived by participants as more of a searching, outcome-oriented task rather than an exploratory one. Nevertheless, interesting outcomes relating to students working within this environment were achieved. The sequel to iFISH (gFISH) facilitates studies that can focus more strongly on user interactions and the role that personal preferences can play in encouraging exploration in playful environments.

\section{ACKNOWLEDGMENTS}

Our thanks to Mitchell Harrop (PhD student at the University of Melbourne) who developed the Flash code for iFISH and gFISH.

\section{REFERENCES}

[1] Adomavicius G., Tuzhilin A. 2005. Toward the Next Generation of Recommender Systems: A Survey of the State-of-the-Art and Possible Extensions. IEEE Transactions on Knowledge and Data Engineering. 17. 734-749.

[2] Carroll J.M., Thomas J.C. 1988. Fun. SIGCHI Bulletin. 19. 21-24.

[3] Chen H., Wigand R.T., Nilan M. 1998. Optimal Flow Experience in Web Navigation. 9th Information Resources Management Association International Conference Effective Utilization and Management of Emerging Information Technologies.
[4] Chen H., Wigand R.T., Nilan M.S. 1999. Optimal Experiences of Web activities. Computers in Human Behavior. 15. 585-608.

[5] Csikszentmihalyi M. 1975. Beyond Boredom and Anxiety. San Francisco: Jossey-Bass Publishers.

[6] Csikszentmihalyi M., LeFevre J. 1989. Optimal Experience in Work and Leisure. Journal of Personality and Social Psychology. 56. 815-822.

[7] Ghani J.A. 1995. Flow in Human-Computer Interactions: Test of a model. In: Carey J.M., ed. Human Factors in Information Systems: Emerging Theoretical Bases. Norwood, New Jersey: Ablex Publishing Corporation, 291-311.

[8] Marchionini G. 2006. Exploratory search: from finding to understanding. Communications of the ACM. 49. 41-46.

[9] Miller S. 1973. Ends, means and galumphing: some leitmotifs of play. American Anthropologist. 75. 87-91.

[10] Pearce J.M. 2004. An investigation of interactivity and flow: student learning during online interaction. $\mathrm{PhD}$ thesis. Department of Information Systems. PhD.

[11] Pearce J.M., Howard S. 2004. Designing for Flow in a Complex Activity. APCHI 6th Asia-Pacific Conference. 349-358.

[12] Pearce J.M., Ainley M., Howard S. 2005. The Ebb and Flow of Online Learning. Computers in Human Behavior. 21. 745-771.

[13] Pearce J.M. 2005. Engaging the Learner: How Can the Flow Experience Support E-learning? E-Learn 2005 Conference.

[14] Pearce J.M. 2008. A System to Encourage Playful Exploration in a Reflective Environment. EdMedia 2008 Conference.

[15] Rieber L.P. 1999. Integrating Web-based Technology into Education: Join the WWILD Team. 1999 Winter Institute for Technology and Adult Basic Education.

[16] Rieber L.P. 2001. Designing learning environments that excite serious play. ASCILITE 2001. 585.

[17] Rieber L.P., Matzko M.J. 2001. Serious Design for Serious Play in Physics. Educational Technology. 41. 14-24.

[18] Salomon G., Globerson T. 1987. Skill may not be enough: The role of mindfulness in learning and transfer. International Journal of Educational Research. 11. 623637.

[19] Starbuck W.H., Webster J. 1991. When is play productive? Accounting, Management and Information Technology. 1.

[20] Stoney S., Oliver R. 1999. Can higher order thinking and cognitive engagement be enhanced with multimedia? Interactive Multimedia Electronic Journal of ComputerEnhanced Learning.

[21] Webster J., Trevino L.K., Ryan L. 1993. The Dimensionality and Correlates of Flow in HumanComputer Interactions. Computers in Human Behavior. 9. 411-426. 